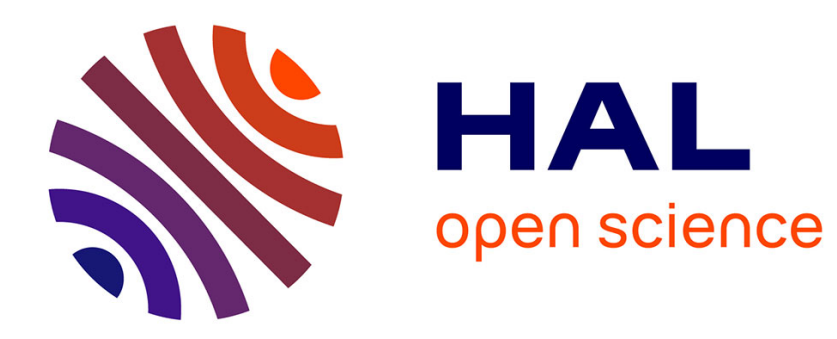

\title{
Le triomphe de "l'europhobie populiste" : surprise électorale ou amnésie française ?
}

\author{
Vincent Geisser
}

\section{To cite this version:}

Vincent Geisser. Le triomphe de "l'europhobie populiste" : surprise électorale ou amnésie française ?. Migrations Société, 2014, 153-154 (3), pp.3. 10.3917/migra.153.0003 . halshs-02113039

\section{HAL Id: halshs-02113039 \\ https://shs.hal.science/halshs-02113039}

Submitted on 29 Apr 2019

HAL is a multi-disciplinary open access archive for the deposit and dissemination of scientific research documents, whether they are published or not. The documents may come from teaching and research institutions in France or abroad, or from public or private research centers.
L'archive ouverte pluridisciplinaire HAL, est destinée au dépôt et à la diffusion de documents scientifiques de niveau recherche, publiés ou non, émanant des établissements d'enseignement et de recherche français ou étrangers, des laboratoires publics ou privés. 
CHERCHER, REPÉRER, AVANCER

\section{LE TRIOMPHE DE “L'EUROPHOBIE POPULISTE” : SURPRISE ÉLECTORALE OU AMNÉSIE FRANÇAISE ?}

Vincent Geisser

Centre d'information et d'études sur les migrations internationales | « Migrations Société »

2014/3 N²153-154 | pages 3 à 10

ISSN 0995-7367

Article disponible en ligne à l'adresse :

https://www.cairn.info/revue-migrations-societe-2014-3-page-3.htm

Distribution électronique Cairn.info pour Centre d'information et d'études sur les migrations internationales.

(c) Centre d'information et d'études sur les migrations internationales. Tous droits réservés pour tous pays.

La reproduction ou représentation de cet article, notamment par photocopie, n'est autorisée que dans les limites des conditions générales d'utilisation du site ou, le cas échéant, des conditions générales de la licence souscrite par votre établissement. Toute autre reproduction ou représentation, en tout ou partie, sous quelque forme et de quelque manière que ce soit, est interdite sauf accord préalable et écrit de l'éditeur, en dehors des cas prévus par la législation en vigueur en France. Il est précisé que son stockage dans une base de données est également interdit. 


\title{
LE TRIOMPHE DE "L'EUROPHOBIE POPULISTE" : SURPRISE ÉLECTORALE OU AMNÉSIE FRANÇAISE ?
}

Vincent GEISSER

\begin{abstract}
«Le Pen dit tout haut ce que tout le monde pense tout bas. C'est ce qu'ont voulu dire les Français et je les en remercie. Ils peuvent me faire confiance. Ce n'est que la première étape d'une longue course qui ira jusqu'à la renaissance française. Ce n'est qu'un début ! ».
\end{abstract}

Jean-Marie Le Pen, soirée électorale à l'occasion des élections européennes, Antenne 2, 17 juin 1984.

«Menace pour la démocratie», «tempête dans la République», «crise sans précédent », «séisme électoral», etc.; les commentateurs et les leaders politiques français ont rivalisé dans l'usage de formules mélodramatiques pour décrire le score soi-disant exceptionnel du Front national (près de $25 \%$ des suffrages ${ }^{1}$ ) aux élections européennes du 25 mai 2014, qui s'affiche désormais sans complexe comme le «premier parti en France ${ }^{2}$.

Sil est vrai que le FN, dans un contexte largement absten-

1. Compte tenu du fait que l'abstention ainsi que les bulletins blancs et nuls ne sont pas pris en compte lors de la proclamation des résultats électoraux, il convient ici de souligner que les pourcentages annoncés sont ceux relatifs aux seules voix exprimées. Or, selon les chiffres officiels du ministère de l'Intérieur (http://elections.intérieur.gouv.fr/ER2014/FE.html), les pourcentages par rapport à la totalité du corps électoral obtenus par les listes arrivées aux cinq premières places sont les suivants : Front national, 10,2 \% ; UMP, 8,47 \% ; PS-MRG $5,69 \%$; Union du centre, 4,05\% ; EE-Les Verts, 3,64\%. Par ailleurs, rappelons que, par rapport à l'élection présidentielle de 2012, le Front national a perdu plus de 1,7 million de voix (4 712 461, contre 6421 426), même si ces pourcentages ont pratiquement quadruplé par rapport aux élections européennes de 2009 pour une abstention légèrement plus faible (57,57 \% contre $59,37 \%$ ) : $10,2 \%$ contre $2,3 \%$ du corps électoral et $24,86 \%$ contre $6,34 \%$ des voix exprimées.

2. LE PEN, Marine, déclaration après l'annonce des premières estimations des résultats des élections européennes, siège du Front national, 25 mai 2014, http://www.nationspresse.info/ fn/europeennes-2014-declaration-de-marine-le-pen-fn 
les couches populaires (ouvriers et employés) et chez les jeunes (moins de 35 ans), il reste sur sa lancée du scrutin municipal de mars $2014^{3}$, relativisant ainsi l'impression de «surprise électorale» qu'aiment cultiver les médias et les politiques français, comme s'ils découvraient, au lendemain du 25 mai, l'ampleur du "phénomène Front national".

Les acteurs politico-médiatiques ont la mémoire courte: cela fait maintenant 30 ans que le Front national confirme son enracinement dans la vie politique française, faisant de l'Hexagone la terre de prédilection de l'extrême droite en Europe. Les scrutins se suivent et se ressemblent, le FN apparaissant depuis de nombreuses années comme une "banalité électorale" dans une France qui continue pourtant à se penser comme une "exception démocratique et républicaine" à l'échelle européenne, pour le meilleur et pour le pire. Qui se souvient, en effet, du score élevé du FN (près de II \%) aux élections européennes de juin 1984 (30 ans déjà!), dans un contexte que l'on qualifiait aussi de «fortement abstentionniste» (43\%)? Le parti lepéniste avait réalisé des scores élevés dans de nombreuses localités méridionales, notamment à Perpignan (21\%), en Corse$\mathrm{du}-\mathrm{Sud}$, dans les Alpes-Maritimes, le Var, les Bouches-du-Rhône (entre $17 \%$ et $22 \%$ ) et surtout à Marseille, cité emblématique, où il était même arrivé en tête, devant le Parti socialiste (21\%, contre I8\%), alors que le premier magistrat de la ville n'était autre que Gaston Defferre, le ministre de l'Intérieur de François Mitterrand ${ }^{4}$.

Mais notre système politique français fonctionne comme une machine à fabriquer de l'amnésie électorale. À chaque fois, les acteurs et les observateurs de la vie publique hexagonale se plaisent à rejouer le scénario du «séisme politique» et de la «surprise électorale» ${ }^{5}$, faisant mine de découvrir la profondeur du phénomène frontiste, avec des explications toutes faites, qui versent le plus souvent dans le registre misérabiliste: la percée électorale de l'extrême droite serait

3. Pour une analyse complète du vote des Français aux élections européennes du 25 mai 2014 voir IPSOS, "Européennes 2014 : comprendre le vote des Français", http://www.ipsos.fr/ ipsos-public-affairs/actualites/2014-05-25-europeennes-2014-comprendre-vote-francais

4. Cf. MOREL, Bernard ; SANMARCO, Philippe, Marseille, l'endroit du décor, Aix-en-Provence : Éd. Édisud, 1985, $191 \mathrm{p}$.

5. DABÈNE, Olivier ; HASTING, Michel ; MASSAL, Julie, La surprise électorale : paradoxes du suffrage universel, Paris : Éd. Karthala ; Aix-en-Provence : Centre de Science Politique Comparative, 2007, $256 \mathrm{p}$. 
l'expression du vote égaré du "petit peuple de France", victime de la crise économique et de la mondialisation ${ }^{6}$. Et tels des pédagogues électoraux ou des maitres d'école de la III ${ }^{\mathrm{e}}$ République, les responsables des principaux partis politiques, soutenus par quelques intellectuels missionnaires ${ }^{7}$, se proposent de ramener le "petit peuple de France" dans le droit chemin, c'est-à-dire le "vote du juste milieu", renvoyant dos à dos l'extrême gauche et l'extrême droite, perçues comme des populismes menaçant la démocratie française. Le quart d'heure d'autoflagellation des politiques («nous sommes responsables du désastre électoral, mais pas coupables») cède rapidement la place à un discours d'autosatisfaction sur les vertus du "modèle républicain", seul capable d'après eux de combattre les extrémistes politiques, auxquels ils ont ajouté, depuis les années 2000, les "radicaux musulmans", même si ces derniers ne disposent pas encore de force électorale ${ }^{9}$.

$\mathrm{T}$ outefois, à y regarder de plus près, cette amnésie électorale (faire fi d'oublier l'implantation ancienne de l'extrême droite dans le paysage politique français) constitue moins un trait psychologique des leaders d'opinion qu'un renvoi à une fonction sociopolitique précise: masquer ou euphémiser la véritable responsabilité des grands partis politiques français dans le processus de banalisation électorale du Front national et, au-delà - phénomène sans doute plus grave - dans le recyclage républicain de ses idées, selon le "théorème de Fabius" énoncé dès le milieu des années i980 et aujourd'hui repris en chœur par un grand nombre de leaders d'opinion: «Le Pen pose les bonnes questions mais donne de mauvaises réponses ${ }^{10}$.

6. Pour une critique salutaire de ce type de thèses, voir COLLOWALD, Annie, Le "populisme du FN" : un dangereux contresens, Bellecombe-en-Bauges : Éd. du Croquant, 2004, 253 p.

7. Cf. FOUREST, Caroline ; VENNER, Fiammetta, Marine Le Pen démasquée, Paris : Éd. Le Livre de Poche, 2012, $480 \mathrm{p}$.

8. Cf. GEISSER, Vincent, "L'intégration républicaine : réflexion sur une problématique postcoloniale", in : BLANCHARD, Pascal ; BANCEL, Nicolas (sous la direction de), Culture postcoloniale 1961-2006 : traces et mémoires coloniales en France, Paris : Éd. Autrement, 2005, pp. 145-164.

9. C'est notamment cette vision binaire qui avait sous-tendu la composition du plateau de l'émission 100 minutes pour convaincre, où Nicolas Sarkozy, alors ministre de l'Intérieur, était opposé à Tariq Ramadan (le "fasciste vert") et à Jean-Marie Le Pen (le "fasciste brun"), France 2, 20 novembre 2013, http://www.ina.fr/video/2169743001

10. Phrase prononcée par Laurent Fabius en 1984, alors qu'il était Premier ministre de François Mitterrand. Ce type de thèses est aujourd'hui repris par l'intellectuel Pierre-André Taguieff. Voir TAGUIEFF, Pierre-André, Le Nouveau national-populisme, Paris : CNRS Éditions, 2012, $128 \mathrm{p}$. 
Il est vrai que depuis 30 ans (1984-2014), les principales forces de gouvernement n'ont cessé de recycler, à des degrés divers, dans leurs programmes électoraux et leurs projets politiques certaines thématiques centrales du Front national sur les questions d'immigration, d'insécurité, d'islam ou de géopolitiques internes et externes, contribuant à brouiller les frontières idéologiques et à légitimer indirectement la "posture frontiste" sur de nombreux problèmes de société. Au fil de nos éditoriaux et contributions parus dans Migrations Société ${ }^{11}$, nous avons largement rendu compte de cette «lepénisation des esprits ${ }^{12}$ qui s'est aussi accompagnée d'une lepénisation des pratiques politiques, influençant de manière significative les politiques publiques en matière d'asile, d'immigration, de nationalité et d'intégration ${ }^{13}$. Le recyclage républicain du discours frontiste a trouvé un prolongement logique dans un mode de gouvernance que nous avons qualifié «d'humanisme sécuritaire ${ }^{14}$, se réclamant simultanément des valeurs des droits de l'homme et du réalisme politique face aux supposés "nouveaux fléaux sociaux" (immigration, islam, communautarisme, etc.).

On trouverait de multiples illustrations de cette gouvernance humanitaro-sécuritaire dans les politiques publiques des gouvernements de droite comme de gauche qui tendraient à prouver que l'extrême droite française n'est pas simplement une force électorale et politique, mais qu'elle est aussi une source d'inspiration idéologique et culturelle qui imprime de plus en plus nos principes et nos valeurs. Pourquoi donc s'étonner de cette "surprise électorale" du Front national le 25 mai 2014, alors que depuis 30 ans (1984: percée

11. Parmi lesquels nous pouvons citer GEISSER, Vincent, "Le Pen Academy, promotion 2007 mots et maux d'une campagne présidentielle", Migrations Société, vol. 19, $\mathrm{n}^{\circ} 110$, mars-avril 2007, pp. 3-15 ; GEISSER, Vincent, "La République est-elle génétiquement modifiable ?", Migrations Société, vol. 19, $\mathrm{n}^{\circ}$ 113, septembre-octobre 2007, pp. 3-18 ; GEISSER, Vincent, Un "anti-tsiganisme" venu d'en haut : le rôle central des élites politiques dans la fabrication du préjugé, Migrations Société, vol. 19, n 109, janvier-février 2007, pp. 107-119 ; GEISSER, Vincent, "Voile identitaire versus francité intégrale : le choc de deux narcissismes communautaires", Migrations Société, vol. 22, n 127, janvier-février 2010, pp. 3-8, etc.

12. TEVANIAN, Pierre ; TISSOT, Sylvie, Dictionnaire de la lepénisation des esprits, Paris : Éd. L'Esprit frappeur, 2002, $373 \mathrm{p}$.

13. Cf. RODIER, Claire ; TERRAY, Emmanuel (sous la direction de), Immigration : fantasmes et réalités, pour une alternative à la fermeture des frontières, Paris : Éd. La Découverte, 2008, $154 \mathrm{p}$.

14. GEISSER, Vincent, “Des 'banlieues de l'Europe' aux banlieues de l'Hexagone : le triomphe de la doctrine de 'l'humanisme sécuritaire"' (éditorial), Migrations Société, vol. 17, n' 102, novembredécembre 2005, pp. 3-11. 
électorale significative du FN lors des élections européennes) les principaux leaders politiques persistent à nier la profondeur du phénomène d'extrême droite dans le paysage politique français (oubliant au passage que la France avait été le pays de naissance $\mathrm{du}$ fascisme contemporain ${ }^{15}$ ) mais contribuent aussi à légitimer "républicainement" ses mots d'ordre nationalistes et sécuritaires à travers la rhétorique politique paradoxale de la dénonciation/ récupération ou, si l'on préfère, de la diabolisation/normalisation?

I est vrai que de son côté, la direction du Front national a accompli un bout de chemin en sens inverse, en cherchant à "lisser" ses thématiques politiques originelles et à gommer les aspects les plus sulfureux de sa propagande idéologique: l'islamophobie a progressivement remplacé l'antisémitisme, la liberté de pensée le négationnisme historique, la complémentarité des sexes (hommes/femmes) le sexisme machiste et la tolérance froide à l'égard de l'homosexualité l'homophobie brutale des pères fondateurs du parti d'extrême droite ${ }^{16}$. Toutefois, ce processus de normalisation du parti lepéniste ne constitue pas simplement un "ravalement de façade" ou une opération de marketing politique en vue de conquérir de nouveaux électorats, mais il renvoie à un processus complexe qui touche plus profondément à la recomposition des principes et des valeurs de l'extrême droite française, comme le relève le politologue Olivier Roy: «Oui, elle [la nouvelle droite] cesse notamment d'être conservatrice sur les questions de famille et de sexualité. Le Front national compte aujourd'hui plus de partisans du mariage homosexuel parmi ses électeurs que l'UMP, si l'on en croit les derniers sondages. Tout simplement parce que ses électeurs sont plus jeunes. La gauche considère la percée de Le Pen comme l'arrivée du fascisme au pouvoir. Or, si le FN est né dans le fascisme, il ne l'est plus, son discours a changé quand la nouvelle droite est arrivée. Aujourd'hui, il n'est plus question du sang, de la terre et des cosmopolites, mais de la culture, des ancêtres et de

15. C'est notamment la thèse défendue par l'historien israélien Zeev Sternhell à laquelle nous souscrivons personnellement. Voir STERNHELL, Zeev Ni droite ni gauche : l'idéologie fasciste en France, Paris : Éd. Fayard, 2000, 537 p.

16. Sur la question du rapport du Front national aux mœurs et aux valeurs, lire l'excellente analyse de CRÉPON, Sylvain, Enquête au ccur du Nouveau Front national, Paris : Nouveau monde éditions, 2012, 302 p. 
l'immigration. C'est plus qu'un “relooking”»" ${ }^{17}$. En ce sens, l'on serait tenté de dire que le Front national s'est autant normalisé par luimême qu'il a été normalisé par les autres forces politiques françaises, selon un processus réciproque.

$\mathrm{Au}$ fur et à mesure que les discours et les pratiques politiques dans l'Hexagone se sont lepénisés, le Front national s'est socialisé à l'univers politique et électoral français, finissant par apparaître comme un "parti comme les autres", en dépit des critiques ponctuelles visant à le stigmatiser. Qui oserait aujourd'hui proposer l'interdiction légale $\mathrm{du}$ Front national au nom du caractère inconstitutionnel et antidémocratique de certains de ses discours et thèmes de campagne? Il faut l'admettre: plus personne ne se risquerait aujourd'hui à réclamer la dissolution du $\mathrm{FN}$, comme certaines personnalités de gauche le revendiquaient dans l'entre-deux-guerres à propos des ligues fascistes. Il est vrai qu'une interdiction produirait davantage d'effets pervers qu'elle ne contribuerait à résoudre le "problème Front national". Mais l'essentiel n'est pas là : le Front national est devenu un parti légitime, parce que ses problématiques idéologiques, ses visions du monde, son décodage des réalités hexagonales apparaissent de plus en plus légitimes aux yeux d'un grand nombre de leaders d'opinion, d'acteurs politiques et médiatiques français. Le "théorème de Fabius" des années 1980 est aujourd'hui pleinement assimilé par les nouveaux élèves de la classe politique française. D'où cette "schizophrénie républicaine" permanente qui consiste simultanément à diaboliser le parti lepéniste pour mieux le normaliser aussitôt, et surtout en lui accordant une "place" au sens premier du terme: le FN a gagné du terrain parce que le système politique français a fini par lui reconnaître un titre de propriété légal sur certaines parcelles. Il est devenu copropriétaire de la "maison France", sans que personne ait rien à y redire. Pire, il apparaît souvent comme le propriétaire le plus légitime, car le plus national, le plus français, le plus authentique, etc.

17. ROY, Olivier, "C'est la fin du discours multiculturaliste en France", interview au journal $L e$ Monde du 29-5-2014, http://www.lemonde.fr/idees/article/2014/05/29/c-est-la-fin-du-discoursmulticulturaliste-en-france_4428420_3232.html 
T e meilleur exemple de cette "schizophrénie républicaine"

Lest justement fourni par la thématique européenne. Comment s'étonner que la campagne europhobe et populiste du FN ait séduit électoralement un quart environ des Français qui se sont déplacés pour déposer dans l'urne un bulletin ni blanc ni nul, quand on sait que la majeure partie des acteurs politiques de l'Hexagone développent eux-mêmes une problématique ambivalente à l'égard de la construction européenne?

Par ailleurs, il faut reconnaître que la plupart des leaders politiques français se sont toujours désintéressés de la consolidation du "principe représentatif" dans l'architecture politique européenne, préférant une construction "par le haut", voire autoritaire ${ }^{18}$, laissant libre cours à toutes les dérives technocratiques et bureaucratiques, entretenant ainsi chez de nombreux Français le sentiment de dépossession politique ou, pire, de dépossession identitaire.

Ce n'est donc pas tant de l'europhobie qui caractérise actuellement les électeurs français qu'une méfiance à l'égard de formes de représentations politiques "froides" et "distanciées", sur lesquelles les citoyens n'auraient plus de prise ${ }^{19}$. Sur ce plan, les leaders politiques français n'ont cessé de délégitimer les critiques sociales de l'Europe, les ringardisant et les assimilant à des manifestations de populisme anti-européen, alors qu'elles exprimaient, au contraire, une volonté de contrôle démocratique et citoyen sur des institutions supranationales. Les partisans d'une "Europe démocratique", qui donnerait davantage de pouvoir au Parlement ${ }^{20}$, et donc aux représentants élus, ont subi autant les foudres de l'extrême droite populiste que celles des principaux partis politiques français (Parti socialiste, UMP, Modem, etc.) qui les ont constamment marginalisés dans le débat public, confortant ainsi le choix réducteur et manichéen: pro-Européens/anti-Européens ou, pire, europhiles/europhobes, sommant les électeurs de choisir entre les deux camps.

18. Cf. MASSARDIER, Gilles, "Les espaces non pluralistes dans les démocraties contemporaines", in : DABENE, Olivier ; GEISSER, Vincent ; MASSARDIER, Gilles (sous la direction de), Démocraties autoritaires, autoritarismes démocratiques au XXF siècle : convergences Nord/Sud, Paris : Éd. La Découverte, 2008, pp. 29-56.

19. Dictionnaire critique de l'Union européenne, Paris : Éd. Armand Colin, 2008, 512 p.

20. Ibidem. 
En outre, la promotion du projet européen a le plus souvent emprunté le registre d'une identité négative, close et défensive, à savoir une Europe qui se protégerait de l'extérieur, des nouvelles invasions barbares: faute d'être capables de construire une Europe pour, les décideurs politiques ont privilégié une Europe contre, par la désignation et la stigmatisation d'ennemis extérieurs, dont l'infiltration sur notre territoire européen est présentée aux opinions publiques comme la menace suprême du XXI ${ }^{e}$ siècle $^{21}$. Plus qu'une idée universaliste, capable de redonner sens à la politique et à la citoyenneté, la vision européenne a été promue par les politiques français, au mieux comme un projet technocratique dénué d'âme démocratique, au pire comme un nouveau rempart pour protéger les "vrais Européens" des tentatives d'incursions étrangères et métèques. On comprend dès lors que le discours europhobe du Front national, qui joue à fond sur les peurs et les crispations anxiogènes, ait pu rafler une grande partie de la mise électorale.

Dlus que l'expression d'un vote antisystème (thème rebattu

1 par les médias et les analystes), le vote frontiste apparaît comme l'émanation logique de l'évolution de notre système politique français qui, depuis plus de 30 ans, a fini par rendre "légitimes" les lectures identitaires des questions sociales. Ainsi, la propension des politiques français à s'étonner des succès électoraux du parti lepéniste apparaît comme une forme d'amnésie volontaire pour mieux occulter leur propre responsabilité dans la banalisation de la posture frontiste.

Beyrouth, le 5 juin 2014

21. Cf. VIANNA, Pedro, "La question migratoire : un enjeu politique pour l'Europe", Migrations Société, vol. $20, \mathrm{n}^{\circ} 116$, mars-avril 2008, pp. 43-57. 